\title{
EVENTO QUEDAS: CUIDADOS DE ENFERMAGEM PARA A SEGURANÇA DO IDOSO HOSPITALIZADO
}

\author{
Anderson Abreu Carvalho ${ }^{1}$ \\ Melissa Orlandi Honório Locks ${ }^{1}$ \\ Silvia Azevedo dos Santos ${ }^{1}$ \\ Angela Maria Alvarez ${ }^{1}$ \\ Karina Silveira de Almeida Hammerschmidt ${ }^{1}$ \\ Jordelina Schier Schier ${ }^{1}$ \\ Darla Lusia Ropelato Fernandez ${ }^{1}$
}

\begin{abstract}
https://orcid.org/0000-0002-4355-1721 https://orcid.org/0000-0003-0972-2053 https://orcid.org/0000-0001-9209-2894 https://orcid.org/0000-0001-9209-2894 https://orcid.org/0000-0002-7140-3427 https://orcid.org/0000-0001-5117-7234 https://orcid.org/0000-0002-6137-7659
\end{abstract}

Objetivo: Identificar os cuidados de enfermagem, quanto ao evento quedas, para a segurança de idosos internados nas unidades de internação de clínica médica. Metodologia: Qualitativa do tipo exploratório-descritivo, realizada no período de março a abril de 2018 , em duas unidades de Clínica Médica de um hospital escola, com 15 enfermeiros. A coleta de dados deu-se através de entrevista semi-estruturada, sendo essas gravadas, transcritas e analisadas segundo análise temática. Resultados: Através das entrevistas identificaram-se três categorias: medidas de prevenção extrínsecas, intrinsecas e comportamentais; cuidados de enfermagem aos idosos que tiveram episódios de quedas e notificação das quedas no ambiente hospitalar. Conclusões: Foi possível concluir que os Enfermeiros conhecem os fatores de risco e implementam ações de prevenção para as quedas, entretanto não conhecem ou pouco utilizam ferramentas e protocolos que padronizem as ações e auxiliem nos cuidados diários ao idoso com risco de quedas.

Descritores: Envelhecimento; Hospitalização; Acidentes por quedas; Cuidados de enfermagem

\section{EVENT FALLS: NURSING CARE FOR THE SAFETY OF THE HOSPITALIZED ELDERLY}

Objective: To identify the nursing care, regarding the event falls, for the safety of elderly hospitalized in the medical clinic hospitalization units. Method: Qualitative of the exploratory-descriptive type, carried out in the period from March to April of 2018, in two units of Medical Clinic of a school hospital, with 15 nurses. Data collection was done through a semi-structured interview, these being recorded. transcribed and analyzed according to Minayo's analysis. Results: Through the interviews, three categories were identified: extrinsic, intrinsic and behavioral prevention measures; nursing care for the elderly who had episodes of falls and notification of falls in the hospital environment. Conclusions: It was possible to conclude that nurses know the risk factors and implement prevention actions for falls, but do not know or use tools and protocols that standardize actions and assist in the daily care of the elderly with risk of falls.

Descriptors: Aging; Hospitalization; Accidents by falls; Nursing care

\section{EVENTO QUEDAS: CUIDADOS DE ENFERMERİA PARA LA SEGURIDAD DEL HOSPITALIZADO}

Objetivo: Identificar los cuidados de enfermería, en cuanto al evento caídas, para la seguridad de ancianos internados en las unidades de internación de clínica médica. Método: Cualitativa del tipo exploratorio-descriptivo, realizada en el período de marzo a abril de 2018 , en dos unidades de Clínica Médica de un hospital escolar, con 15 enfermeros. La recolección de datos se dio a través de entrevista semiestructurada, siendo esas grabadas, transcritas y analizadas según análisis de Minayo. Resultados: A través de las entrevistas se identificaron tres categorías: medidas de prevención extrínsecas, intrínsecas y comportamentales; cuidados de enfermería a los ancianos que tuvieron episodios de caídas y notificación de las caidas en el ambiente hospitalario. Conclusiones: Fue posible concluir que los enfermeros conocen los factores de riesgo e implementan acciones de prevención para las caídas, sin embargo no conocen o poco utilizan herramientas y protocolos que estandaricen las acciones y auxilien en los cuidados diarios al anciano con riesgo de caídas.

Descriptores: Envejecimiento; hospitalización; Accidentes por caídas; Cuidados de enfermería

${ }^{1}$ Universidade Federal de Santa Catarina.

Autor Correspondente: Anderson Abreu Carvalho - Email: anderson.imbitubal2@gmail.com 


\section{INTRODUÇÃO}

O processo de envelhecer é influenciado por diversos fatores, entre eles as doenças crônicas e os eventos adversos onde se destaca o evento quedas. A ocorrência de quedas é um indicador da perda da postura e da alteração de marcha, estando relacionada à insuficiência súbita de mecanismos neurais e osteomioarticulares ${ }^{(1)}$.

As quedas têm como definição, a ida a uma superfície de contato, a um nivel inferior ao que estava anteriormente, sendo este um contato não intencional, resultante da perda de equilibrio postural, sem que tenha havido um acidente inevitável ou perda de consciência ${ }^{(2)}$. As mesmas podem ser originadas tanto por fatores intrínsecos, como as alterações fisiológicas do envelhecimento, doenças crônicas e a polifarmácia; quanto por fatores extrínsecos, geralmente os quais são decorrentes de circunstâncias socioambientais. O reconhecimento da multifatorialidade associada às quedas auxilia no esclarecimento dos fenômenos causais e consequentemente, nas medidas preventivas ${ }^{(3)}$.

As quedas são frequentes no ambiente hospitalar. podendo representar $70 \%$ dos eventos adversos e dois de cada cinco eventos indesejáveis relacionados à segurança do paciente. Índices indicam que de cada 1000 pacientes internados, 1,1 a $22 \%$ sofrem quedas nos ambientes hospitalares ${ }^{(4)}$. As quedas de pacientes internados estão entre os principais eventos a serem prevenidos nas instituições e são prioridades dentro do programa de segurança do paciente. Elas podem acarretar consequências graves, do tipo: traumas teciduais, fraturas, retirada não programada de artefatos terapêuticos e morte $^{(5)}$.

No Brasil, as quedas vêm se destacando entre as causas externas com grande impacto no perfil de mortalidade na população e ampla relevância na morbidade(6). Portanto, é mister que o Enfermeiro saiba identificar os fatores de riscos para poder prevenir e posteriormente tratar as eventuais consequências das quedas.

\section{OBJETIVO}

Identificar os cuidados de Enfermagem, quanto ao evento quedas, para a segurança de idosos internados nas unidades de internação de clínica médica.

\section{MÉTODO}

\section{Tipo de estudo}

Trata-se de uma pesquisa qualitativa, do tipo exploratório-descritiva.

\section{Participantes do estudo}

Participaram 15 Enfermeiros de duas unidades de internação que atenderam aos critérios de inclusão de estar atuando há seis meses na instituição e como critérios de exclusão, os Enfermeiros que não realizavam assistência direta ao paciente.

\section{Local do estudo}

Realizado em duas unidades de Clínica Médica de um Hospital de ensino em Florianópolis/SC

\section{Coleta dos dados}

Foi realizada no período de março a abril de 2018 através de entrevista semi-estrutura contendo perguntas abertas referentes à caracterização dos sujeitos, além de perguntas atinentes aos temas fatores de riscos, avaliação, prevenção e cuidados dispensados ao idoso que tenham quedas no ambiente hospitalar, com duração média de 30 minutos.

\section{Procedimentos de análises dos dados}

Os dados oriundos das entrevistas foram transcritos e analisados à luz da análise temática de Minayo, seguindo as etapas de pré-análise; exploração do material e, por fim, o tratamento e interpretação dos resultados, de onde emergiram os temas e subtemas referentes às unidades de significados emergentes.

\section{Procedimentos éticos}

Projeto aprovado pelo Comitê de Ética em Pesquisa com Seres Humanos da Universidade Federal de Santa Catarina, CAAE: 80907517.5.0000.0121. Todos os participantes assinaram o Termo de Consentimento Livre e Esclarecido.

\section{RESULTADOS}

Participaram 15 Enfermeiros, sendo seis da Clínica Médica 1 e nove da Clínica Médica 2, onde 14 eram mulheres e um homem e idade variando entre 26 e 54 anos; 14 estão formados a mais de oito anos; todos os entrevistados possuem especialização; 10 possuem Mestrado. Quanto ao tempo de trabalho na unidade, oito há menos de 1 ano; seis de 1 a 10 anos e um a mais de 20 anos.

Após a análise das falas advindas das entrevistas emergiram três categorias temáticas e subtemas conforme Figura 1. 
Figura 1: Temas e subtemas oriundos das entrevistas com enfermeiros de duas Clínicas Médicas de um hospital de ensino de Florianópolis. Florianópolis, SC, Brasil, 2018

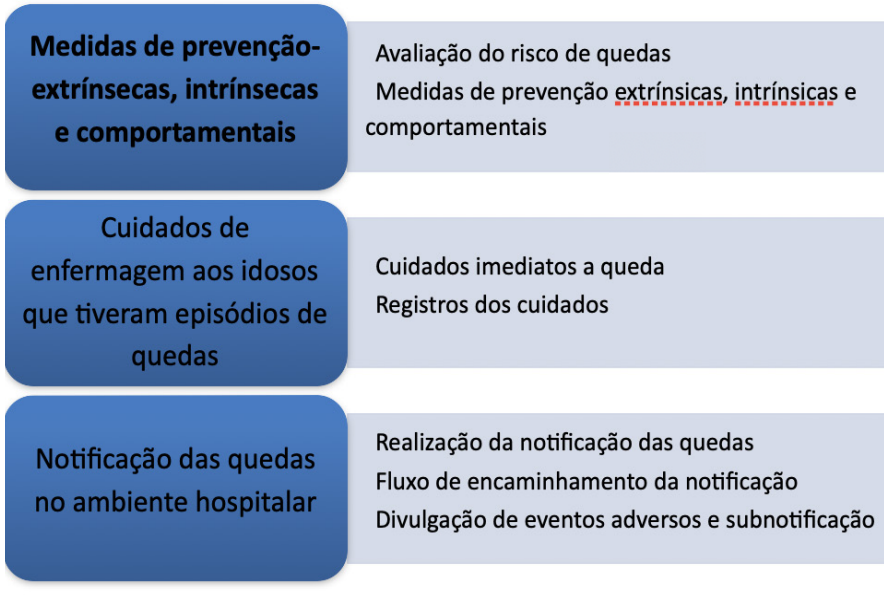

Medidas de prevenção extrinsecas, intrinsecas e comportamentais

$\mathrm{Na}$ questão sobre prevenção de quedas no ambiente hospitalar, os enfermeiros verbalizaram não seguir uma rotina pré-estabelecida de avaliação do risco de quedas na admissão do paciente, uma vez que a mesma se dá através de uma avaliação subjetiva do paciente, sem a utilização de escalas ou instrumentos. O que pode ser observado na emissão a seguir:

Uma rotina pré-estabelecida, não tem. O que já é rotina, é essa avaliação do paciente quanto à mobilidade, sua parte neurológica, se precisa de andador, apoio para se locomover, orientação do uso de dispositivo de mobilidade ou nosso auxílio. (E15)

No que se refere à identificação da necessidade de medidas de prevenção, esta ocorre em momentos distintos, como na passagem de visita nos leitos e na avaliação direta ao paciente. Alguns ainda colocam que registram no prontuário e que também fazem orientações aos pacientes:

[...] avaliar da melhor forma possivel os riscos do paciente, a gente prescreve na prescrição de enfermagem e coloca na evolução...temos esse cuidado de colocar a prevenção de quedas e os cuidados específicos. (E3)

Dentre os fatores de risco, foi possivel identificar nas falas que os mesmos identificam diversas situações relacionadas aos riscos para quedas, como os fatores extrínsecos, intrínsecos e comportamentais. Neste sentido,
14 entrevistados falaram como prioridade nas ações de prevenção dos fatores extrínsecos a elevação das grades do leito e acompanhar os pacientes nas atividades de vida diárias:

[... ] a cama estar alta, as vezes ele não abaixa para descer, e tenta sair da cama ainda com ela alta. O ambiente inadequado também é um risco. Não temos escadinha em todos os leitos. O Piso escorregadio [...]. (El)

Paciente agitado, desorientado e sem acompanhante temos que manter na contenção mecânica.(E2)

Nos fatores intrínsecos, as falas mais evidentes foram em relação a alguns medicamentos que causam alterações neurológicas, tontura, sonolência e hipotensão, além da polifarmácia:

[...] as vezes ele está com hipoglicemia, hipotensão postural, vertigem, uma série de fatores que temos que avaliar, para que ele possa sair da cama com segurança. $(E l)$

Temos que observar, as medicações que causam tonturas, para dar uma maior atenção. A própria polifarmácia é um risco.(E2)

No que tange aos fatores de riscos comportamentais, as questões verbalizadas foram em relação às ações e atividades de vida diária e o uso de dispositivos para locomoção como cadeira de rodas, bengala, chinelos anti-derrapantes entre outros:

[...] orientar de trazer um chinelo fechado, ou o solado do sapato de borracha, na hora do banho. Se apoiar nas barras .... o cuidado no banho, para não escorregar.(El3)

Em relação à escala de avaliação do risco de quedas (Morse) utilizada na instituição, 11 entrevistados sabem que tem a referida escala padronizada na unidade e quatro nunca ouviram falar. Do total de entrevistados, oito utilizam a escala para a avaliação em seus cuidados cotidianos.

Cuidados de enfermagem aos idosos que tiveram episódios de quedas

Como ações de cuidados às quedas, os pesquisados destacaram a orientação e a educação em saúde, tanto voltada aos pacientes quanto para a equipe de enfermagem: 
A orientação dos riscos que ele está correndo, se ele sair sozinho do leito, tiver passando mal e de repente não está aqui no setor. Orientar de acordo com a condição dele. (El)

Em relação às ações de cuidados imediatos aos idosos que tiveram quedas, os entrevistados elencaram realizar diversas ações, dentre elas a avaliação neurológica, da dor, verificar a presença de ferimento ou fratura e identificação das causas. Outros cuidados específicos foram mencionados, porém os 15 entrevistados verbalizaram a chamada do plantão para avaliação mais amiúde do paciente. As informações sempre são registradas no prontuário:

[...] primeiramente verifico se ele está consciente tem alguma dor, algum machucado aparente, caso não, enquanto uma pessoa está dando assistência eu já peço para que o outro já chame o plantão. Registro no prontuário. (El)

Diante da ocorrência de quedas no ambiente hospitalar, faz-se necessária a notificação do evento para que a instituição conheça o panorama e planeje estratégias para prevenção.

\section{Notificação das quedas no ambiente hospitalar}

No que consiste a notificação das quedas, apenas quatro verbalizaram não saber o fluxo das notificações, porém 11 verbalizaram fazer a notificação, três não os fazem e um mencionou não saber quanto à necessidade de notificar as quedas:

Eu não sabia que tinha... eu não sabia que era notificado... não conheço a ficha, não sei como se faz. (El)

[...] eu sei que quem retira a notificação da unidade é - COSEP e eles fazem uma estatística e a partir disso eles baseiam as ações [...]. (E6)

Em relação à devolutiva do relatório interno aos enfermeiros que notificaram, 12 enfermeiros não sabem e/ou não viram uma devolutiva das notificações e três demonstraram conhecer o fluxo:

As vezes vem verificar as causas, colhem dados nos prontuários, verificam os resultados das ações e conversam sobre o que pode mudar... os dados são divulgados no sistema do HU. (E15)

[...] até hoje eu não vejo uma devolutiva. (E4)
Outro dado importante, foi que 11 enfermeiros falaram da subnotificação dos eventos quedas na instituição:

Tentam entender porque aconteceu ... não tenho capacitação sobre quedas... nem conhecimento sobre a divulgação dos dados... mas são subnotificadas [...]. (E13)

\section{DISCUSSÃO}

Sumariamente, percebe-se nas falas que são desenvolvidos cuidados como forma de prevenir as quedas, porém os mesmos não são padronizados.

No Brasil, uma investigação realizada sobre quedas de idosos hospitalizados em um hospital universitário do sul do Brasil, evidenciou que, em cada 1000 leitos, cerca de três a $17 \%$ dos indivíduos caem por dia ${ }^{(8)}$. Neste sentido, os enfermeiros entrevistados dizem não seguir uma rotina sistematizada de avaliação dos riscos de quedas sendo essa, realizada de forma subjetiva, uma vez que não utilizam instrumentos para essa avaliação. A enfermagem é de extrema importância na assistência à saúde do idoso em todos os níveis. Para tal, utiliza como método para a concretude organizacional do cuidado, a Sistematização da Assistência de Enfermagem, que se dá através do Processo de Enfermagem ${ }^{(9)}$. Os registros são apontados como um grande aliado na assistência, tendo em vista que eles servem de parâmetro frente às ações que foram realizados e também no gerenciamento das atividades ${ }^{(10)}$

Especificamente sobre os riscos de quedas, os entrevistados elencaram aspectos referentes a três tipos existentes: riscos extrínsecos, intrínseco e comportamentais. Os achados desse estudo corroboram com outro, onde apontam que as principais intervenções de Enfermagem para a prevenção de quedas em ambientes hospitalares são: elevar grades do leito, campainha perto do leito, barras de segurança pelo corredor e banheiro, piso antiderrapante, boa iluminação entre outras intervenções, como avaliar o risco de quedas e os fatores ambientais na admissão do paciente ${ }^{(11)}$.

No que se refere à prevenção foi mencionado também o uso de contenção mecânica em pacientes agitados, desorientados e sem acompanhantes. A esse respeito, a Resolução do Conselho Federal de Enfermagem N. 427 (2012), que normatiza o uso de contenção mecânica empregado por Enfermeiros, profere que a mesma deverá ser empregada quando for o único meio disponível para prevenir um dano imediato e não será por um período prolongado, respeitando sempre as liberdades individuais e o espírito humanístico do cuidado ${ }^{(12)}$

Em relação aos riscos intrínsecos, os entrevistados elencaram o uso e os efeitos das medicações e patolo- 
gias associadas. Neste sentido, o estudo aponta que os fatores de riscos relacionados às medicações, as que mais influenciam nas quedas são, os psicotrópicos, ansiolíticos e cardiovasculares ${ }^{(13)}$. Dentre outros fatores intrínsecos destaca-se as alterações sensório motoras, em que o indivíduo fica mais debilitado advindo do processo saúde doença e a dificuldade propiciada pelo ambiente desconhecido ${ }^{(14)}$

Uma pesquisa realizada em Porto alegre revela que cerca de $72 \%$ dos idosos apresentam pelo menos uma doença crônica, necessitando do tratamento farmacológico e do uso regular de fármacos, facilitando a polifarmácia ${ }^{(15)}$. Uns dos principais problemas da terapia medicamentosa no idoso, porque são mais susceptiveis aos efeitos colaterais e as funções de diversos órgãos tornam-se deficientes, modificando a atividade dos fármacos ${ }^{(16)}$.

No que se refere aos riscos comportamentais, é necessário que sejam observadas as peculiaridades de cada idoso, atentando para o uso de dispositivos de marcha, vestimentas e calçados inadequadas, uso de álcool, entre outros fatores ${ }^{(17)}$

Umas das formas de sistematizar o cuidado de maneira científica e baseado em evidências é através da construção de protocolos, pois, evitam equívocos ${ }^{(18)}$. A utilização de protocolos mais focados no cuidado à prevenção de quedas e a identificação dos fatores de risco, tem se apresentado como uma alternativa bastante eficiente. Essa mediação tem refletido de maneira positiva na qualidade de vida dos idosos e tem se tornado ferramenta essencial para o enfermeiro no processo de melhoria do atendimento(19).

As principais estratégias de cuidados imediatos aos idosos que caíram no ambiente hospitalar, que mais apareceram no estudo, foram orientação e educação em saúde aos idosos. Neste sentido, a utilização de estratégias de educação dos pacientes e familiares deve incluir orientações sobre o risco de queda, consequências e prevenção. Essas ações devem ocorrer na admissão e durante a permanência do paciente no hospital ${ }^{(20)}$

Em relação aos cuidados imediatos dispensados aos pacientes após as quedas, indica-se a realização da supervisão periódica para avaliação da segurança do paciente, além de avaliar sempre o nível de consciência. Outros cuidados ainda são sugeridos, como: orientar o paciente e acompanhante para somente levantar do leito acompanhado por profissional; avaliar o nível de dependência para planejamento da assistência relacionado à mobilização, autonomia para deambulação e a necessidade de utilização de dispositivo de marcha(21)
No que se refere à notificação das quedas, identificou-se uma subnotificação das mesmas. Nesse sentido, sabe-se que o processo de educação permanente dos profissionais de saúde para a notificação é gradativo, até que esteja no dia a dia da realidade vivida. É importante o investimento na disseminação da cultura de segurança, com ênfase em educação e comunicação, adoção de condutas baseadas em protocolos, para ampliar o conhecimento em segurança ${ }^{(20)}$.

O enfermeiro deve sempre depois de ocorrido um evento adverso registrar o período do dia em que ocorreu, registrar as causas, fatores predisponentes, a conduta imediata e as consequências, registrando as intervenções realizadas, bem como a notificação ${ }^{(21)}$.

\section{Limitações do estudo}

Como limitação do estudo configura-se o fato de ter sido realizado apenas com Enfermeiros. Sugere-se assim, que estudos que envolvam outros membros da equipe de e-Enfermagem sejam de grande valia, uma vez que são eles que diretamente realizam o cuidado diário ao idoso.

\section{Contribuições para a prática}

Esse estudo possibilitou a identificação dos fatores de risco, avaliação e manejo das quedas em idosos hospitalizados. Ao mapear este panorama do cuidado, possibilita-se aos Enfermeiros e à instituição criar estratégias através do desenvolvimento de ações e tecnologias que possibilitem a mudança da prática, com vistas a aprimorar a Sistematização da Assistência de Enfermagem e a segurança do idoso, minimizando as quedas e suas complicações no ambiente hospitalar.

\section{CONSIDERAÇÕES FINAIS}

Foi possivel identificar que os enfermeiros realizam ações de cuidados, através do conhecimento dos fatores de riscos e da implementação das ações de prevenção para as quedas em idosos e ações de cuidados imediatos, entretanto não conhecem ou pouco utilizam ferramentas que padronizem as ações e auxiliem nos cuidados diários ao idoso com risco de quedas, bem como a notificação.

Acredita-se que essa pesquisa no cenário da prática, possibilitou realizar um panorama das ações de cuidado realizadas aos idosos, podendo servir como base para estudos futuros no que se refere à padronização de cuidados a este segmento da população, estimulando também o desenvolvimento de estratégias de educação permanente à equipe de enfermagem. 


\section{AGRADECIMENTOS}

Agradecimentos ao PROCAD/CAPES, cujo presente estudo faz parte, estando vinculado ao Macroprojeto "Envelhecimento Ativo: promoção da saúde e prevenção de incapacidades funcionais e cognitivas".

\section{Contribuição dos Autores:}

Concepção e/ou desenho: AAC, MOHL, SAS, JS, AMA, KSAH; Análise e Interpretação dos dados: AAC, MOHL, SAS, JS, AMA, KSAH; Redação do artigo: AAC, MOHL, SAS; Revisão crítica: AAC, MOHL, SAS; Revisão Final: AAC, MOHL, SAS, JS, AMA, KSAH.

\section{REFERÊNCIAS}

1 Mendes TAB. Geriatria e Gerontologia. 4aㅡ ed. São Paulo: Editora Manole; 2017.

2 Abreu HCA, Reiners AAO, Azevedo RCS, Silva AMC, Abreu DROM, Oliveira AD. Incidence and predicting factors of falls of older inpatients. Rev Saúde Pública [Internet]. 2015. p. 49:37 Acessado em: 11 de jan de 2019;. Disponivel em: http://www.scielo.br/pdf/rsp/v49/ 0034-8910-rsp-S0034-89102015049005549.pdf

3 Souza LHR, Brandão JCS, Fernandes AKC, Cardoso BLC. Quedas em idosos e fatores de riscos associados. Rev. Aten. Saúde, São Caetano do Sul (Internet), v.15, n.54. 2017. p.55-60. Acessado em: 10 de jan de 2019. Disponivel em: http://seer.uscs.edu.br/index.php/revista_ciencias_saude/article/view/4804/pdf

4 Vieira ER, Berean C, Paches D, Caveny P, Yuen D, Ballash L, et al. Reducing falls among geriatric rehabilitation patients: a controlled clinical trial. Clin Rehabil. [Internet]. 27(4). 2013; p. 325-35. Acessado em 14 de jan de 2019: 27(4): 325-35. Disponivel em: www.ncbi. nlm.nih.gov/pub$\mathrm{med} / 22952303$

5 Brito TA, Fernandes MH, Coqueiro RS, Jesus CS. Quedas e capacidade funcional em idosos longevos residentes em comunidade. Texto Contexto - Enferm. [Internet] 2017; 22(1) [acesso em 21 abr 2017]. Disponivel: http://dx.doi.org/10.1590/S0104-07072013000100006.

6 Alves GCQ, Silva AD, Ohl RIB, Amaral EMS, Ferreira LA, Chavaglia SRR. Vitimas de trauma por quedas atendidas em unidades de pronto socorro adulto: estudo transversal. Rev. Enferm. em Foco (Internet) 2018, 9(3), p.59-65. Disponivel em: http://revista.cofen.gov.br/index.php/enfermagem/article/view/1958/462. Acessado em: 14 de jan de 2019.

7 Luzia MF, Cassola TP, Suzuki LM, Dias VLM, Pinho LB, Lucena AF. Incidência de quedas e ações preventivas em um Hospital Universitário. Rev. Esc. Enferm. USP. (Internet) 2018; 52 (3). Acessado em 14 de jan de 2019. Disponivel em: https://www.revistas.usp.br/reeusp/article/ view/145282/139378.

8 Melo DFF, Nunes TAS, Viana MRP. Percepção do enfermeiro sobre a implantação da sistematização da assistência de enfermagem no centro cirúrgico. R. Interd. [Internet] 2014; 2(7) [acesso em 23 jul 2018]. Disponivel: https://revistainterdisciplinar.uninovafapi.edu.br/index.php/revinter/article/view/425/pdf_126.

9 Silva DT, Goulart NS, Amado KC. Registros de enfermagem com ênfase na segurança do paciente. Revista Rede de Cuidados em Saúde. 2014; 8(2)

10 Alshammari AS, Alhassan AM, Aldawsari MA, Bazuhair FO, Alotaibi FK, Aldakhil AA et al. Falls among elderly and its relation with their health problems and surrounding envitonmental factors in riyadh. $J$ Family Community Med. 2018 jan-apr; 25(1):29-34
11 Conselho Federal de Enfermagem. Resolução n. 427, de 07 de maio de 2012. Normatiza os procedimentos da enfermagem no emprego de contenção mecânica de pacientes. Brasilia: COFEN; 2012.

12 Instituto para práticas seguras no uso de medicamentos (ISMP) Medicamentos associados a riscos de quedas. 2017. [acesso em 21 abr 2018]. Disponivel: http://www.ismp-brasil.org/site/wpontent/uploads/2017/02/IS_0001_17_Boletim_Fevereiro_ISMP_210x276mm.pdf

13 Paixão CMJ, Heckman MF. Distúrbios da postura, marcha e quedas. Tratado de Geriatria e Gerontologia. 4 ed. Rio de Janeiro: Guanabara Koogan; 2017.

14 Silva AR, Sgnaolin V, Nogueira EL, Loureiro F, Engroff P, Gomes I. Doenças crônicas não transmissiveis e fatores sociodemográficos associados a sintomas de depressão em idosos. 2017; 66(1), p. 45-51. Acessado em: 14 de jan de 2019. Disponivel em: http://www.scielo.br/ pdf/jbpsiq/v66nl/0047-2085-jbpsiq-66-1-0045.pdf

15 Silva EA, Macedo LC. Polifarmácia em idosos. Revista Saúde e Pesquisa. [Internet] 2013; 6(3) [acesso em 24 abr 2018]. Disponivel: http:// dx.doi.org/10.17765/1983-1870.2013v6n3p\%25p.

16 Falsarella GR, Gasparotto LPR, Coimbra AMV. Quedas: conceitos, frequências e aplicações à assistência ao idoso. Rev. Bras. Geriatr. Gerontol, [Internet] 2014; 17(4) [acesso em 21 abr 2017]. Disponivel: http:// dx.doi.org/10.1590/1809-9823.2014.13064.

17 Porto KLHA. A segurança do paciente na utilização do checklist. Rev. Enfermagem Revista. [Internet] 2014; 17(2) [acesso em 23 jul 2018]. Disponivel: http://periodicos.pucminas.br/index.php/enfermagemrevista/article/view/12876/10087.

18 Petito ADC, Costa LFX, Petito G. Souza FAA. Prevenção de quedas em idosos a partir do autocuidado: uma revisão bibliográfica. REFACER. [Internet] 2015; 4(2) [acesso em 23 jul 2018]. Disponivel: http:// ceres.facer.edu.br/revista/index.php/refacer/article/view/88/57.

19 Ministério da Saúde (BR). Portaria n. 529, 1ㅇ de abril de 2013. Institu o Programa Nacional de Segurança do Paciente (PNSP). Diário Oficial [da] República Federativa do Brasil. 2013 abr. 02; Seção 1. p 43. Brasil. Constituição da República Federativa do Brasil. Brasilia: Senado; 2013. 20 Siman AG, Cunha SGS, Brito MJM. A prática de notificação de eventos adversos em um hospital de ensino. Rev. esc. enferm. USP. [Internet] 2017; (51) [acesso em 22 abr 2018]. Disponivel: São Paulo, v.51. Out-2017. Disponivel em:http://dx.doi.org/10.1590/s1980$220 \times 2016045503243$.

RECEBIDO: 05/12/2018

ACEITO: 23/9/2019 